Service social

\title{
Multi-Jeunesse : une expérience d'entraide par les pairs
}

\section{Marie-Claude Michaud et Johanne Voyer}

Volume 35, numéro 3, 1986

Les jeunes et le travail social

URI : https://id.erudit.org/iderudit/706315ar

DOI : https://doi.org/10.7202/706315ar

Aller au sommaire du numéro

Éditeur(s)

École de service social de l'Université Laval

ISSN

1708-1734 (numérique)

Découvrir la revue

Citer cet article

Michaud, M.-C. \& Voyer, J. (1986). Multi-Jeunesse : une expérience d'entraide par les pairs. Service social, 35(3), 316-322. https://doi.org/10.7202/706315ar
Résumé de l'article

La possibilité de développer une approche d'entraide par les pairs pour intervenir avec des jeunes en planification des naissances et en sexualité intéresse de nombreux professionnels. Un projet d'entraide (projet "

Multi-Jeunesse ») en milieu scolaire secondaire et collégial a été mis sur pied à Rimouski, en août 1983. Le questionnement constant et les différentes techniques utilisées ont permis de développer certaines pistes d'intervention. Quelle est la conception de l'entraide véhiculée ? Quels objectifs d'intervention ont été privilégiés? Quelles méthodes pratiques ont été développées dans les groupes avec les jeunes ? Voici les thèmes qui font l'objet de cet article. 
Michaud, Marie-Claude, intervenante au projet Multi-Jeunesse.

VOYER, Johanne, intervenante au projet Multi-Jeunesse.

\section{Multi-jeunesse : une expérience d'entraide par les pairs}

\section{Marie-Claude Michaud Johanne Voyer}

Depuis plusieurs années, la Clinique de planification des naissances du Département de santé communautaire du Centre hospitalier régional de Rimouski intervient, entre autres, auprès des jeunes. La connaissance des principales problématiques reliées à leur vécu et l'intérêt à développer des approches nouvelles répondant davantage aux besoins de cette clientèle ont conduit à la conceptualisation du projet MultiplicateursJeunesse. Appelé plus communément Multi-Jeunesse, ce programme de prévention vise les objectifs suivants: diminuer la fréquence des grossesses non désirées chez les adolescentes, abaisser l'incidence des maladies transmissibles sexuellement, réduire le délai existant entre le début des relations sexuelles, l'utilisation de la contraception et améliorer le vécu sexuel des adolescents. La particularité de ce projet se situe au niveau du type d'intervention qui a été retenu pour l'atteinte de ces objectifs, soit l'expérimentation de l'approche d'entraide par les pairs.

En effet, dans le cadre d'une subvention accordée par la Direction de la promotion de la santé au Département de santé communautaire du Centre hospitalier de Rimouski, Multi-Jeunesse a permis de faire l'expérience d'une approche qui vise à former de jeunes multiplicateurs pouvant aider d'autres jeunes, comme eux, dans leur vécu par rapport à la sexualité. Ainsi, d'août 1983 à juillet 1986, une intervention a eu lieu dans deux écoles : à la polyvalente Paul-Hubert et au Cégep de Rimouski. Elle a rejoint exclusivement une clientèle d'élèves âgés d'environ 14 à 21 ans, de niveaux secondaire III, IV, $\mathrm{V}$ et collégial.

Dans cet article, on pourra prendre connaissance de la conception de l'entraide telle que véhiculée dans le projet, des objectifs privilégiés, ainsi que de l'articulation concrète du programme. 


\section{La conception de l'entraide dans le programme Multi-Jeunesse}

L'esprit du programme est de favoriser, chez les jeunes impliqués, le développement de relations d'aide naturelles plutôt que de relations d'aide inspirées du modèle professionnel.

Ainsi, on ne vise pas à transformer les multiplicateurs en experts de la relation d'aide, mais plutôt à les amener à développer et à raffiner des attitudes d'entraide qui respectent ce qu'ils sont déjà. Il faut reconnaître que les jeunes n'ont pas tous à aider de la même façon ; la jeunesse ne correspond pas à un tout homogène, mais plutôt un mélange de styles différents, d'individus distincts qui vivent et communiquent entre eux à leur manière. Nous avons la conviction que la majorité des jeunes ont du potentiel pour s'entraider : chacun le fera différemment, mais un jeune d'un certain style sera sûrement la personne la plus appropriée pour intervenir auprès d'un autre qui présente le même style que lui. Par exemple, Lucie et Diane, deux étudiantes impliquées dans MultiJeunesse, ont deux genres bien différents. Lucie est très souvent à la bibliothèque de l'école, elle y côtoie ses amis et les rencontre aussi dans des activités sportives. Diane, elle, ne vit pas du tout dans les mêmes lieux et se retrouve toujours avec son groupe de motards. Nul besoin d'insister sur le fait que ces deux multiplicatrices ne s'expriment pas du tout de la même façon et que, dans des situations d'entraide, elles réagissent bien différemment. Toutefois, chacune s'avère la personne la plus influente et la plus adéquate pour diffuser de l'information dans son propre groupe d'amis. Elles connaissent bien leurs pairs, ont le même langage, une même manière d'être et d'agir. Une attitude d'ouverture envers les différents styles de jeunes a permis, jusqu'à présent, de rejoindre un éventail plus large de réseaux d'étudiants. Reconnaissons toutefois que l'intervention quotidienne demande beaucoup de souplesse afin d'adapter le programme en fonction de cette clientèle variée.

Afin de distinguer aide et entraide, précisons que la première réfère à une action individuelle alors que la seconde s'intéresse au caractère réciproque de ce type d'action, très présent chez les jeunes. On essaie de démystifier le terme entraide pour le ramener à un geste simple du quotidien, qui peut se faire n'importe où et n'importe quand. Par exemple, David informe Louis que les condoms sont disponibles en pharmacie sans prescription. De son côté, Hélène, à la suite d'une rencontre Multi-Jeunesse où on a parlé de réactions sexuelles, suscite une discussion enflammée durant son party d'anniversaire. Enfin, Julie 
rappelle à Sébastien que la Clinique de dépistage des maladies transmissibles sexuellement (M.T.S.) est ouverte en avant-midi seulement. Voilà quelques situations vécues qui illustrent bien que l'entraide signifie tout autant multiplier des informations autour de soi qu'apporter une assistance concrète à un individu dans une situation précise.

\section{Les objectifs}

À partir de cette conception de l'entraide, voyons plus spécifiquement les quatre objectifs privilégiés dans notre expérience.

1. favoriser l'acquisition de connaissances en planification des naissances et en sexualité (par exemple : l'approche gars-filles, les relations sexuelles, la contraception, etc.);

2. faire connaître et susciter l'utilisation des services existants;

3. développer des habiletés à la communication et à l'entraide ;

4. développer quelques habiletés pour l'organisation et l'animation de certaines activités dans le milieu.

Pour clarifier davantage ces quatre objectifs, précisons d'abord que le programme ne cherche pas à former des "superspécialistes» de la sexualité. On vise plutôt à ce que les jeunes acquièrent un certain bagage de connaissances qui puisse correspondre à leur capacité d'intégration. En effet, des connaissances peu intégrées sont vite oubliées par les multiplicateurs et ont peu de chance d'être diffusées auprès de leurs pairs.

On insiste de plus sur une bonne connaissance des services existants, rejoignant ainsi le deuxième objectif, qui vise à faire connaître les ressources pour obtenir une information manquante, orienter ou référer un ami, etc. À ce propos, le programme de formation pour les multiplicateurs inclut toujours une activité où ils sont invités tout spécialement à visiter la Clinique de planification des naissances et la Clinique de dépistage des M.T.S. du Centre hospitalier de Rimouski.

En ce qui concerne le troisième objectif, tel que précisé plus haut, on ne donne pas une formation intensive et spécifique sur la relation d'aide. Toutefois, les activités visent une certaine croissance personnelle des jeunes (entre autres, au niveau de la connaissance de soi, de l'expression de soi et de la confiance en soi) et le développement d'attitudes aidantes comme l'écoute, le respect, la compréhension.

Finalement, pour atteindre le dernier objectif, les multiplicateurs sont invités, durant le programme, à participer à l'animation des rencontres ainsi qu'à la création et à l'organisation d'activités plus spéciales offertes à l'ensemble des étudiants de l'école contactée. Soulignons, entre 
autres, la réalisation d'un mini-colloque sur la sexualité au Cégep et la tenue d'un « midi-spécial » d'information à la Polyvalente.

\section{Le déroulement}

Voyons maintenant comment le programme se concrétise. Au début de la session scolaire, dans chacune des deux écoles choisies, on amorce une période de recrutement et différents moyens sont utilisés pour publiciser les dates d'inscription.

Après trois années d'expérimentation, le projet est mieux connu. Les étudiants ayant déjà participé à Multi-Jeunesse en parlent régulièrement à leurs amis, ce qui augmente le nombre de candidats intéressés à adhérer au projet. Ainsi, il devient parfois nécessaire de procéder à une certaine sélection des candidats puisqu'un maximum de deux à trois groupes par milieu, comprenant 8 à 12 participants, sont formés à chaque semestre.

Quoique certains critères d'adhésion soient considérés, aucune grille d'évaluation formelle n'est utilisée pour sélectionner les multiplicateurs. Les plus jeunes sont choisis d'abord puisque leur présence dans le milieu scolaire devrait vraisemblablement être plus longue. De plus, par quelques questions, on évalue l'intérêt et la motivation par rapport au projet.

Principalement, les jeunes sont formés lors d'une série de rencontres hebdomadaires, huit à dix par session, pour chacun des groupes. Ces réunions durent environ deux heures et demie. Le programme de formation est structuré de façon à ce qu'ils puissent examiner un thème différent à chaque semaine. Les contenus sont définis en fonction des groupes et de leurs besoins. La programmation n'est donc pas toujours établie à l'avance, avant le début de la session; toutefois, certains sujets jugés essentiels comme des notions d'anatomie, le cycle menstruel, la contraception, etc., sont régulièrement discutés.

L'élaboration d'un plan de rencontres privilégie la façon dont le thème est abordé et les exercices qui s'y rattachent, car ces deux éléments sont déterminants pour la réussite du programme. En ce sens, on a une préoccupation constante de voir à ce que le contenu des rencontres soit traité de manière dégagée, à partir d'activités vivantes et toujours différentes.

Ainsi, à titre d'exemple, précisons que les réunions débutent toujours par une " prise de contact ", i.e. une activité qui vise à permettre à chaque jeune d'exprimer comment il se sent au début de la rencontre. Le dessin, individuel ou collectif, le " thermomètre de mon humeur ", le "graphique de mon moral ", etc., sont quelques-uns des outils que nous 
avons créés et que nous utilisons à cette fin. Bref, il s'agit, par là, d'amorcer la rencontre de façon joyeuse et vivante et d'exprimer ce que l'on vit dans une forme qui soit un peu hors du commun. Viennent ensuite les exercices qui, chaque semaine, permettent de discuter le thème de la sexualité qu'on a prévu traiter plus en profondeur. Encore là, il faut toujours trouver des façons originales de le faire. Par exemple, lors de discussions en petits groupes, des présentations libres sur la contraception peuvent donner lieu à la visite simulée d'un vendeur de condoms, à l'enseignement d'un médecin, à la tenue d'un "quizzcontraception ». Une autre fois, c'est à partir d'un dessin géant de sa propre silhouette, réalisé avec une note humoristique, qu'on discutera de certaines notions d'anatomie. Notons qu'il reste beaucoup de place pour l'imprévu et la créativité des jeunes, ainsi que pour l'expression de leur vécu durant l'exercice.

On insiste également pour que ces activités se déroulent dans un esprit d'entraide ; celle-ci est d'abord une réalité qui se vit globalement dans le projet et non seulement à l'extérieur auprès des amis. Ainsi d'anciens multiplicateurs sont invités à participer à l'intégration des nouveaux dans le groupe et à partager avec ceux-ci les connaissances déjà acquises.

Finalement, le moment de l'évaluation clôture toujours une rencontre Multi-Jeunesse. En effet, pour nous, il est primordial de permettre aux jeunes de s'exprimer sur ce qu'ils y ont vécu. Ce moment permet d'aller recueillir les commentaires des étudiants, en vue d'évaluer et de rajuster l'intervention. Parfois, aussi c'est un instant privilégié où, en fin de session, on posera un geste plus spécial d'amitié et de solidarité comme, par exemple, un repas communautaire.

Ajoutons aussi qu'il est nécessaire, dans un tel cadre d'intervention, d'entretenir entre les rencontres de formation un contact avec les jeunes dans le but d'alimenter leur motivation et de les supporter dans leurs gestes d'entraide quotidiens.

\section{Les intervenants impliqués et leur rôle}

Les principales intervenantes, auteures de cet article, travaillent toutes les deux à temps complet auprès de jeunes en milieu scolaire. L'équipe comprend aussi un éducateur-sexologue à temps partiel, un contractuel chargé de l'évaluation du processus et de l'impact du projet ainsi qu'un médecin de la Clinique de planification des naissances lequel est responsable de son déroulement.

La tâche spécifique des intervenantes consiste à assumer la réalisation concrète du projet dans les écoles et, plus précisément, à concevoir et à 
structurer un programme de formation qui, à chaque session du calendrier scolaire, s'adresse à des groupes de jeunes désireux de devenir des multiplicateurs.

\section{Commentaires}

À ce moment-ci de l'expérimentation, les jeunes démontrent une grande satisfaction face aux expériences que Multi-Jeunesse leur permet de vivre : ils disent apprendre beaucoup. De plus, on peut observer quotidiennement les gestes d'entraide que posent les jeunes entre eux. Le type d'intervention que nous avons développé demande beaucoup d'énergie, de souplesse et de créativité. Toutefois, en utilisant avec les jeunes une approche d'égal à égal, nous y vivons, là aussi, des relations très enrichissantes, pleines d'authenticité et de plaisir. Notre première attitude est d'être à l'écoute des jeunes; à partir de là, nous inventons et nous nous questionnons. Les trois années d'expérimentation bientôt terminées, un rapport d'évaluation exhaustif sera réalisé. On pourra sûrement y puiser des pistes d'intervention. Toutefois, nous croyons que toute expérience de ce genre doit s'adapter au milieu, et que la meilleure piste à suivre demeure celle qui nous vient des jeunes euxmêmes.

\section{Références bibliographiques}

Arseneau, J., C. Bouchard, M. Bourgon, G. Goupil, J. Guay, F. Lavoie et R. Perreault, Psychothérapies: Attention !, Québec, Québec Science éditeur, 1983.

Bérubé, J., G. LÉGare, M.-C. Michaud et J. Voyer, "Éducation sexuelle en milieu scolaire ", Le médecin du Québec, vol. 20, n 11, 1985 : 83-87.

BOWMAN, R.P. et R.D. MYRICK, "I'm a junior counselor, having lots of fun", The School Counselor, vol. 28, no 1, septembre $1980: 31-38$.

CARr, R.A., Le co-conseil, théorie et pratique, exposé présenté au cours de la consultation nationale sur l'orientation professionnelle, Ottawa, Commission de l'emploi et de l'immigration du Canada, 1981.

De Pietro, R., "Can adolescents be motivated to learn about birth control ? ", International Quarterly of Community Health Education, vol. 3, no 4, 19821983 : 337-350.

Fournier, F. et M. Blais, Styles et valeurs des jeunes, Montréal, Bureau de consultation jeunesse inc., 1984.

GuAY, J., L'intervenant professionnel face à l'aide naturelle, Chicoutimi, Gaëtan Morin, 1984. 
NADEAU, B., L'entraide chez les jeunes : perspectives d'intervention et de recherche, allocution présentée au colloque de la Fédération des centres locaux de services communautaires sur l'approche communautaire auprès des 0-18 ans, Montréal, 1983.

TESSIER, M., Sexualité et prévention: d'abord l'affaire des jeunes, Montréal, Bureau de consultation jeunesse inc., 1985.

THORNBURG, H.D., "Adolescent sources of information on sex ", Journal of School Health, vol. 51, no 4, avril 1981: 274-277. 